\title{
MODIFIED COSMOLOGICAL EQUATIONS AND THE EINSTEIN STATIC UNIVERSE
}

\author{
LUCA PARISI AND ROSANGELA CANONICO
}

Communicated by Ivaïlo M. Mladenov

\begin{abstract}
The stability properties of the Einstein Static solution of General Relativity is altered when corrective terms arising from modifications of the underlying gravitational theory appear in the cosmological equations. Employing dynamical system techniques and numerical integrations, we discuss the stability of static cosmological solutions in the framework of two recently proposed quantum gravity models, namely Loop Quantum Cosmology and Horava-Lifshitz gravity.
\end{abstract}

\section{Introduction}

The Einstein Static (ES) Universe is an exact solution of Einstein's equations describing a closed Friedmann-Robertson-Walker model sourced by a perfect fluid and a cosmological constant (see, for example [23]). This solution is unstable to homogeneous perturbations as shown by Eddington [15], furthermore it is always neutrally stable against small inhomogeneous vector and tensor perturbations and neutrally stable against adiabatic scalar density inhomogeneities with high enough sound speed [2].

In recent years there has been renewed interest in the ES Universe because of its relevance for the Emergent Universe scenario [16, 17,31] in which the ES solution plays a crucial role, being an initial state for a past-eternal inflationary cosmological model. In the Emergent Universe scenario the horizon problem is solved before inflation begins, there is no singularity, no exotic physics is involved, and the quantum gravity regime can even be avoided. This model, relying on the choice of a particular initial state, suffers from a fine-tuning problem which is ameliorated when modifications to the cosmological equations arise but then a mechanism is needed to trigger the expanding phase of the Universe (see [27,28]).

The existence of ES solutions along with their stability properties has been widely investigated in the framework of General Relativity for several kinds of matter fields sources (see [3] and references therein). ES solutions also exist in several modified gravity models [8] ranging from the Randall-Sundrum and DGP 
braneworld scenarios $[12,18,22,37,42]$ to Gauss-Bonnet modified gravity and $f(R)$ theories [4-6, 13, 20, 21,36]. The issue of the existence and stability of ES solutions has also been considered in the semiclassical regime of Loop Quantum Cosmology (LQC), in either the case of correction to the matter sector [32] or the case of correction to the gravitational sector [34]. Recently the same issue has been also considered in the framework of Hořava-Lifshitz (HL) gravity [41] and IR modified Hořava gravity [7, 19].

When dealing with higher order modified cosmological equations, the existence of many new ES solutions is possible, whose stability properties, depending on the details of the single theory or family of theories taken into account, are substantially modified with respect to the classical ES solution of General Relativity (GR).

Often in such analysis the case of closed $(k=1)$ cosmological models is the only one considered, neglecting the intriguing possibility of static solutions in open $(k=-1)$ cosmological models. It is interesting that, due to the aforementioned corrections to the cosmological equations, open ES models may be found even in the case of a vanishing cosmological constant or when the perfect fluid has vanishing energy density.

In this paper we systematically review the stability properties of static cosmological solutions arising in the framework of two recently proposed quantum gravity models, namely Loop Quantum Cosmology and Horava-Lifshitz gravity, both providing modified cosmological equations. To this aim, we employ dynamical system techniques and numerical integrations. This work is based on the results presented in $[10,33,34]$.

This paper is structured as follows. In Section 2, we consider static solutions in the framework of LQC, following and enlarging the analysis already performed in [34]. It is shown that, beside the ES solution of GR, a LQC solution arises also in the case of open cosmological models which stability is also completely characterized. Following the same approach, in Section 3 we consider static cosmological solutions in the context of HL gravity with detailed balance and projectability condition. Two solutions are found along with their stability properties. In Section 4, some conclusions are eventually drawn.

\section{Loop Quantum Cosmology}

In Loop Quantum Cosmology the quantization techniques borrowed by Loop Quantum Gravity, a background-independent nonperturbative quantum theory of gravity, are applied to symmetry reduced models (see [9] and references therein). 
For the sake of simplicity, in this section we consider the modified Friedmann equations arising in the semiclassical regime of LQC [1,39]. We consider gravitational modifications only, neglecting the inverse volume correction to the matter sector. The motivation is twofold: the analysis of this system allows a more transparent comparison with the case of GR and moreover it allows us to follow the notations introduced in [34] which will also be easily used in the analysis of the HL gravity presented in the next section.

The model considered is sourced by a perfect fluid with linear equation of state $p=$ $w \rho$ plus a cosmological constant $\Lambda$. The classical energy conservation equation still holds

$$
\dot{\rho}=-3 \rho H(1+w)
$$

while the loop quantum effects lead to a modification to the classical Friedmann equation

$$
H^{2}=\left(\frac{\kappa}{3} \rho+\frac{\Lambda}{3}-\frac{k}{a^{2}}\right)\left(1-\frac{\rho}{\rho_{c}}-\frac{\Lambda}{\kappa \rho_{c}}+\frac{3 k}{\kappa \rho_{c} a^{2}}\right)
$$

and to the Raychaudhuri equation

$$
\begin{aligned}
\dot{H}= & -\frac{\kappa}{2} \rho(1+w)\left(1-\frac{2 \rho}{\rho_{c}}-\frac{2 \Lambda}{\kappa \rho_{c}}\right) \\
& +\left[1-\frac{2 \rho}{\rho_{c}}-\frac{2 \Lambda}{\kappa \rho_{c}}-\frac{3 \rho(1+w)}{\rho_{c}}\right] \frac{k}{a^{2}}+\frac{6 k^{2}}{\kappa \rho_{c} a^{4}} .
\end{aligned}
$$

Notice that we are considering at once the $k=0$ case and the $k= \pm 1$ cases [1,39]. Here $\kappa=8 \pi G=8 \pi / M_{P}^{2}$, and the critical LQC energy density is $\rho_{c} \approx 0.82 M_{P}^{4}$.

\subsection{Static Solutions}

The system of equations (1)-(4) admits two static solutions, i.e. solutions characterized by $\dot{a}=\dot{H}=\dot{\rho}=0$. The first solution corresponds to the standard ES Universe in GR while the second solution arises from the LQC corrective terms

$$
\begin{aligned}
\rho_{G R}=\frac{2 \Lambda}{\kappa(1+3 w)}, & a_{G R}^{2}=\frac{2 k}{\kappa \rho_{G R}(1+w)} \\
\rho_{L Q}=\frac{2\left(\Lambda-\kappa \rho_{c}\right)}{\kappa(1+3 w)}, & a_{L Q}^{2}=\frac{2 k}{\kappa \rho_{L Q}(1+w)} .
\end{aligned}
$$

The conditions under which these static solutions exist are summarized in Table 1 which follow from $a^{2}>0$ and $\rho>0$. The presence of the curvature index $k$ is worth stressing, indeed the previous analysis [34] can be enlarged to enclose the $k=-1$ case where the two solutions still exist. 


\subsection{Stability Analysis}

The stability of the solutions equations (4) and (5) can be characterized using dynamical system theory and performing a linearized stability analysis. To this aim, we first have to rewrite the system of equations (1)-(4) in the form of a genuine dynamical system. Indeed, in these equations the three variables $a, H$ and $\rho$ appear but the actual dynamics is constrained on a two-dimensional surface described by the modified Friedmann equation (see Fig.1). Thus, following [34], we solve

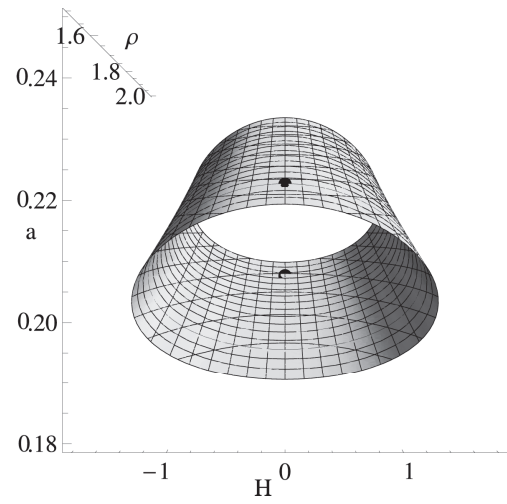

Figure 1. Friedmann constraint as hypersurface in the $a, H, \rho$ space the for the case $k=-1, \Lambda<0, w<-1$ with $\Lambda=-100, w=-2$, $\kappa=25.13274123$. The ES and LQ solutions are depicted as black dots on top and underneath the surface respectively.

equation (2) for $1 / a^{2}$. Two solutions are found

$$
\frac{1}{a^{2}}=g_{ \pm}(\rho, H)
$$

where

$$
g_{ \pm}=\frac{2(\kappa \rho+\Lambda)+\kappa \rho_{c}\left(1 \pm \sqrt{1-12 H^{2} / \kappa \rho_{c}}\right)}{6 k}
$$

Substituting the solutions (6) into equation (4), we find two branches for the time derivative of the Hubble parameter, thus the original system splits in a pair of twodimensional nonlinear dynamical systems in the variables $\rho$ and $H$ (see Fig. 2)

$$
\begin{array}{lll}
\text { GR }: \dot{\rho}=-3 H \rho(1+w) & \text { and } & \dot{H}=F_{-}(\rho, H) \\
\text { LQ }: \dot{\rho}=-3 H \rho(1+w) & \text { and } & \dot{H}=F_{+}(\rho, H)
\end{array}
$$


where

$$
\begin{aligned}
F_{ \pm}= & -\frac{\kappa}{2}(1+w) \rho\left(1-\frac{2 \rho}{\rho_{c}}-\frac{2 \Lambda}{\kappa \rho_{c}}\right)+\frac{6 k^{2} g_{ \pm}^{2}}{\kappa \rho_{c}} \\
& +g_{ \pm} k\left[1-\frac{2 \rho}{\rho_{c}}-\frac{2 \Lambda}{\kappa \rho_{c}}-3(1+w) \frac{\rho}{\rho_{c}}\right] .
\end{aligned}
$$

Each one of the systems (8) and (9) admits a fixed point representing a static solution, that is

$$
\begin{aligned}
& \mathrm{GR}: H=0 \quad \text { and } \quad \rho_{o}=\frac{2 \Lambda}{\kappa(1+3 w)} \\
& \mathrm{LQ}: H=0 \quad \text { and } \quad \rho_{o}=\frac{2\left(\Lambda-\kappa \rho_{c}\right)}{\kappa(1+3 w)}
\end{aligned}
$$

respectively. Substituting these values of $\rho_{O}$ in equation (2) one gets exactly the values of the constant scale factor in terms of the parameters as in equations (4) and (5).

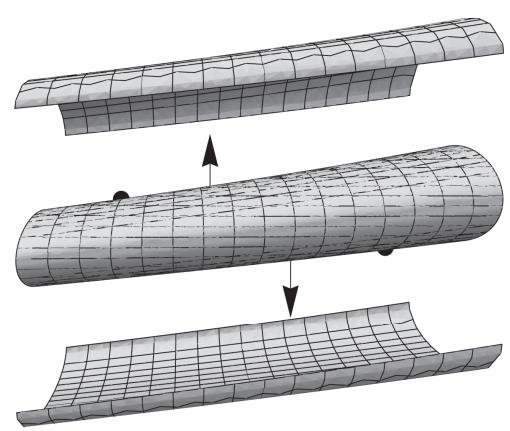

Figure 2. Splitting of the Friedmann constraint in two local charts around the fixed points.

Finally, to characterize the stability of the solutions equations (4) and (5) we evaluate the eigenvalues of the Jacobian matrix for the two systems equations (8) and (9) at the fixed points equations (11) and (12) respectively.

For the system in equation (8), we recover the usual properties of the ES solution in GR. The eigenvalues of the linearized system at the fixed point are

$$
\lambda_{G R}= \pm \sqrt{\Lambda(1+w)}
$$

In the case of positive curvature index $k=1$, these are either real with opposite signs for $\Lambda>0$ and $w>-1 / 3$ - thus the fixed point is unstable (of the saddle 
type) - or purely imaginary for $\Lambda<0$ and $-1<w<-1 / 3$, so the fixed point is a center. In the case of negative spatial curvature index $k=-1$, these are again real with opposite signs for $\Lambda<0$ and $w<-1$, so the fixed point is unstable (of the saddle type). In Fig. 3 an example of the latter case is depicted.

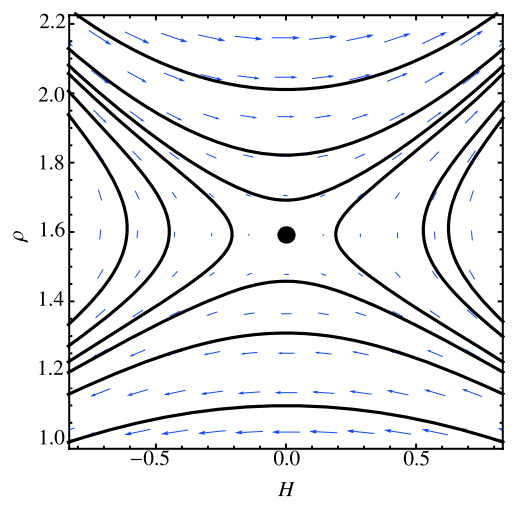

Figure 3. Dynamical behavior of the system around the GR fixed point for the case $k=-1, \Lambda<0, w<-1$ with $\Lambda=-100, w=-2$, $\kappa=25.13274123$.

For the system equation (9) the eigenvalues at the fixed point are

$$
\lambda_{L Q}= \pm \sqrt{\left(\kappa \rho_{c}-\Lambda\right)(1+w)} .
$$

In the case of positive curvature index $k=1$, the LQ fixed point is either unstable (of the saddle kind), when $\kappa \rho_{c}>\Lambda$ and $-1<w<-1 / 3$, or a center for the linearized system, i.e. a neutrally stable fixed point, when $\kappa \rho_{c}<\Lambda$ and $w>-1 / 3$. In the case of negative spatial curvature index $k=-1$, the eigenvalues are purely imaginary for $\kappa \rho_{c}>\Lambda$ and $w<-1$, so we have a center for the linearized system again. In the latter case the fixed point is nonhyperbolic thus the linearization theorem does not apply. Nevertheless a numerical integration of the fully nonlinear system equation (9) for initial conditions near the fixed point confirms the result of the linearized stability analysis (see Fig. 4). It's worth stressing that in open LQC models a stable ES solution exists in the case of positive values of the cosmological constant as long as $\Lambda<\kappa \rho_{c}$.

The results of the linearized stability analysis are summarized in Table 1. 


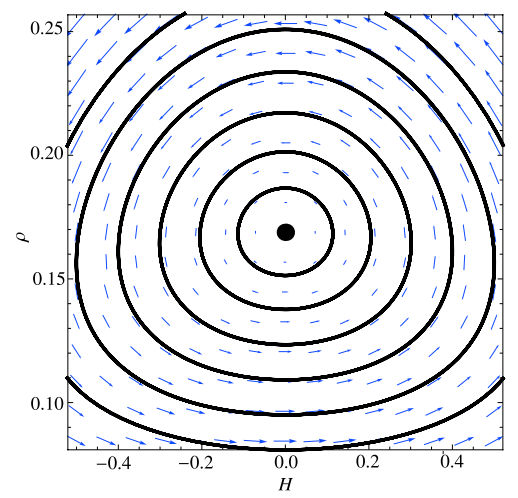

Figure 4. Dynamical behavior of the system around the LQ fixed point for the case $k=-1, \Lambda<\kappa \rho_{c}, w<-1$ with $\Lambda=10, w=-2, \kappa=25.13274123$.

Table 1. Existence conditions and stability conditions for the static solutions in equations (4) and (5).

\begin{tabular}{|c|c|c|c|c|}
\hline & $\mathrm{k}$ & $\Lambda$ & $w$ & Stability \\
\hline \multirow{3}{*}{ GR } & 1 & $>0$ & $w>-1 / 3$ & saddle \\
\cline { 3 - 5 } & & $<0$ & $-1<w<-1 / 3$ & center \\
\cline { 2 - 5 } & -1 & $<0$ & $w<-1$ & saddle \\
\hline \multirow{2}{*}{ LQ } & \multirow{2}{*}{1} & $<\kappa \rho_{c}$ & $-1<w<-1 / 3$ & center \\
\cline { 3 - 5 } & & $>\kappa \rho_{c}$ & $w>-1 / 3$ & saddle \\
\cline { 2 - 5 } & -1 & $<\kappa \rho_{c}$ & $w<-1$ & center \\
\hline
\end{tabular}

\section{Hořava-Lifshitz Gravity}

The Hořava-Lifshitz gravity $[24,25]$ is a power-counting renormalizable theory of (3+1)-dimensional quantum gravity. In the ultraviolet limit, the theory has a Lifshitz-like anisotropic scaling between space and time characterized by the dynamical critical exponent $z=3$. In the IR limit the theory flows to the relativistic value $z=1$.

The effective speed of light $c$, the effective Newton constant $G$ and the effective cosmological constant $\Lambda$ of the low-energy theory, emerge from the relevant deformations of the deeply nonrelativistic $z=3$ theory which dominates at short distances [24, 25]

$$
c=\frac{\kappa^{2} \mu}{4} \sqrt{\frac{\Lambda_{W}}{1-3 \lambda}}, \quad G=\frac{\kappa^{2}}{32 \pi c}, \quad \Lambda=\frac{3}{2} \Lambda_{W} .
$$


The first of the equations in(15) imposes a relation among the parameters $c, \Lambda_{W}$ and $\lambda$; thus, in order to have a real emergent speed of light $c$, for $\lambda>1 / 3$ the cosmological constant has to be negative $\Lambda_{W}$. However, after an analytic continuation of the parameters (see [29]), a real speed of light for $\lambda>1 / 3$ implies a positive cosmological constant $\Lambda_{W}$. Thus, mimicking the notation introduced in [30], we introduce a two-valued parameter $\epsilon= \pm 1$, in order to examine both the aforementioned cases at once.

The HL cosmology has been systematically studied using dynamical systems theory in $[11,14,26,38]$, it has also been investigated in [40] using conservation laws of mechanics. Here we consider static solutions of the cosmological equations for the HL gravity when both the detailed balance condition and projectability condition hold.

First we recast the modified Friedmann equations of [29] in a form which allows an easy comparison with the formerly considered case of LQC ${ }^{1}$.

The modified Friedmann equation reads

$$
H^{2}=\frac{2}{3 \lambda-1}\left[\frac{\kappa}{3} \rho+\epsilon\left(\frac{\Lambda}{3}-\frac{k}{a^{2}}+\frac{3 k^{2}}{4 \Lambda a^{4}}\right)\right]
$$

and the modified Raychaudhuri equation reads

$$
\dot{H}=\frac{2}{3 \lambda-1}\left[-\frac{\kappa}{2} \rho(1+w)+\epsilon\left(\frac{k}{a^{2}}-\frac{3 k^{2}}{2 \Lambda a^{4}}\right)\right] .
$$

The conservation equation for the energy density of the perfect fluid still holds unchanged:

$$
\dot{\rho}=-3 \rho H(1+w) .
$$

Besides the overall factor $\frac{2}{3 \lambda-1}$ on the right hand side of equations (16) and (17), the modifications to the cosmological equations of GR consist of the higher order terms $\propto k^{2} / \Lambda a^{4}$ which become dominant at short distance scales and do not affect the classical cosmological equations in the case of flat models.

\subsection{Static Solutions}

It can be readily found, imposing the conditions $\dot{a}=\dot{H}=\dot{\rho}=0$, that the system of equations (18)-(17) admits the following two static solutions

$$
\begin{aligned}
\rho_{H L 1} & =0, & a_{H L 1}^{2} & =\frac{3 k}{2 \Lambda} \\
\rho_{H L 2} & =\frac{-16 \epsilon \Lambda}{(3 w-1)^{2} \kappa}, & a_{H L 2}^{2} & =\frac{(3 w-1) k}{2 \Lambda(1+w)} .
\end{aligned}
$$

\footnotetext{
${ }^{1}$ According to the definitions given in Section II, $c=1$ and $\kappa=8 \pi G$, equation (16) and equation (17) have been written accordingly.
} 
The conditions under which these static solutions exist are summarized in Table 2 and Table 3.

The presence of the curvature index $k$ and the parameter $\epsilon$ in equations (19) and (20) is worth being stressed; indeed the analysis presented in [41] can be enlarged to enclose the $k=-1$ case where new interesting possibilities arise. For instance a physically meaningful ES solution is present even in the case of vanishing energy density of the perfect fluid, i.e., equation (19).

\subsection{Stability Analysis}

The stability analysis can be easily performed reducing the original system to an actual two-dimensional autonomous dynamical system by making use of the Friedmann constraint (see Fig.5). In this case the simplest and most straightforward

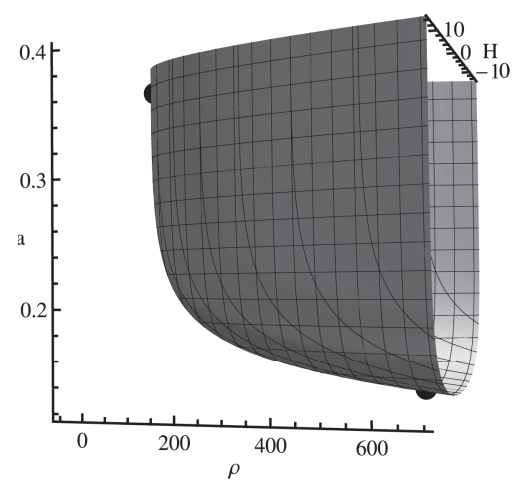

Figure 5. Friedmann constraint as hypersurface in the $a, H, \rho$ space the for the case $k=-1$ with $\epsilon=1, \lambda>1 / 3, \Lambda<0, w>1 / 3$. The two black dots represent the HL1 (upper) and HL2 (lower) static solutions.

choice is to eliminate the dependence on $\rho$ from the other equations, being equation (16) linear in $\rho$, that is, to consider the projection on the $(H, a)$-plane (see Fig.6). This allows us to describe the dynamics with just one set of equations. Indeed, solving equation (16) for $\rho$

$$
\rho=\frac{3}{2 \kappa}(3 \lambda-1) H^{2}-\frac{\epsilon}{\kappa}\left(\Lambda-\frac{3 k}{a^{2}}+-\frac{3 k^{2}}{4 \Lambda a^{4}}\right)
$$

and substituting into equation (17) one gets a first order nonlinear differential equation

$$
\dot{H}=\frac{\epsilon}{3 \lambda-1}\left[(1+w) \Lambda-\frac{(3 w+1) k}{a^{2}}+\frac{3 k^{2}(3 w-1)}{4 \Lambda a^{4}}\right]-\frac{3}{2}(1+w) H^{2}
$$


which, together with the definition of the Hubble parameter

$$
\dot{a}=a H
$$

provides a genuine two-dimensional autonomous dynamical system in the variables $a$ and $H$. The system admits two fixed points with energy densities as in equations (19) and (20) and in order to characterize the stability of these solutions, we evaluate the eigenvalues of the Jacobian matrix for the system equations (22) and (23) at the fixed points corresponding to equations (19) and (20) respectively.

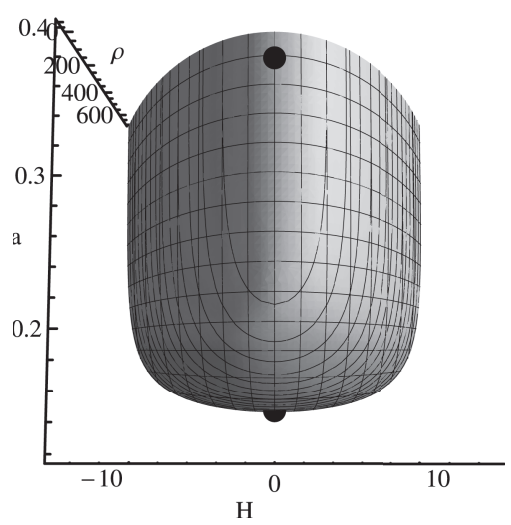

Figure 6. Friedmann constraint as seen from the $(H, a)$-plane.

The eigenvalues at the fixed point $H L 1 \mathrm{read}$

$$
\lambda_{H L 1}= \pm \frac{2 \sqrt{6(3 \lambda-1) \epsilon \Lambda}}{3(3 \lambda-1)} .
$$

For all the admitted values of the parameters this is a pair of purely imaginary eigenvalues thus the fixed point is a center for the linearized system. The point is nonhyperbolic so the linearized analysis may fail to be predictive at nonlinear order, nevertheless a numerical integration proves that this fixed point is actually a center (see Fig. 7).

The results of the stability analysis for the fixed point $H L 1$ are summarized in Table 2.

The eigenvalues at the fixed point $H L 2$ read

$$
\lambda_{H L 2}= \pm \frac{2 \sqrt{-2(3 w-1)(3 \lambda-1)(1+w) \epsilon \Lambda}}{(3 \lambda-1)(3 w-1)} .
$$




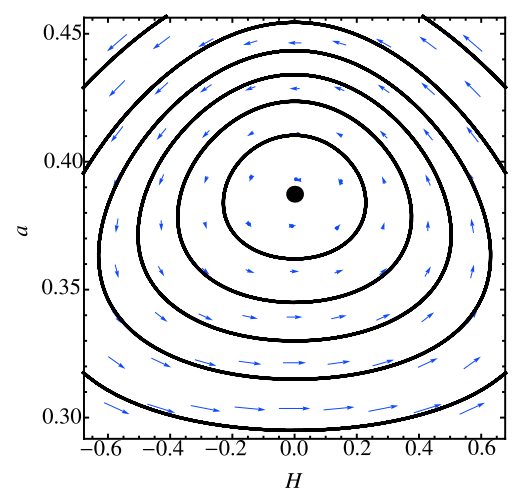

Figure 7. Dynamical behavior of the system around the $H L 1$ fixed point for the case $k=-1$ with $\epsilon=1, \lambda>1 / 3, \Lambda<0, w>1 / 3$.

Table 2. Existence conditions and stability conditions for the static solution $H L 1$.

\begin{tabular}{|c|c|c|c|c|}
\hline$\epsilon$ & $\lambda$ & $k$ & $\Lambda$ & Stability \\
\hline \multirow{3}{*}{-1} & $<1 / 3$ & -1 & $<0$ & \multirow{2}{*}{ center } \\
\cline { 2 - 4 } & $>1 / 3$ & 1 & $>0$ & \multirow{1}{*}{} \\
\hline \multirow{2}{*}{1} & $<1 / 3$ & 1 & $>0$ & \multirow{1}{*}{} \\
\cline { 2 - 4 } & $>1 / 3$ & -1 & $<0$ & \multicolumn{1}{|c}{} \\
\cline { 2 - 4 } & & &
\end{tabular}

According to the admitted values of the parameters this is either a pair of purely imaginary eigenvalues, so the fixed point is a center for the linearized system, or a pair of real eigenvalues with opposite signs, so the fixed point is unstable (of the saddle type). In particular, the solution is a center for $-1<w<1 / 3$ and is a saddle for $w<-1$ or $w>1 / 3$ (for an example of the latter case see Fig. 8).

The results of the stability analysis for the fixed point $H L 2$ are summarized in Table 3.

\section{Conclusions}

Here we have considered the existence of static solutions in the framework of two recently proposed quantum gravity models, namely, LQC and HL gravity and eventually we have shown that the inclusion of a negative curvature index $k=-1$ enlarges the ranges of existence of the solutions affecting their stability properties 


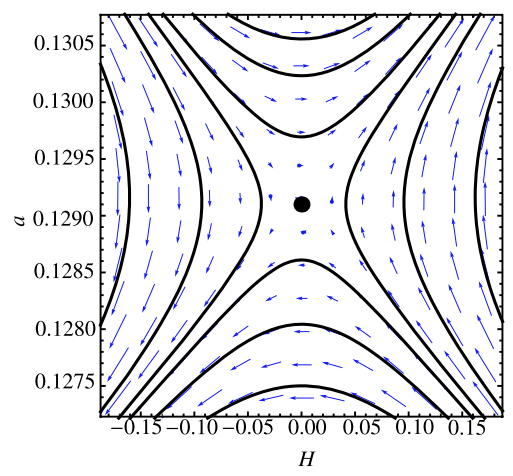

Figure 8. Dynamical behavior of the system around the $H L 2$ fixed point for the case $k=-1$ with $\epsilon=1, \lambda>0, \Lambda<0, w>1 / 3$.

Table 3. Existence conditions and stability conditions for the static solution HL2.

\begin{tabular}{|c|c|c|c|c|c|}
\hline$\epsilon$ & $\lambda$ & $k$ & $\Lambda$ & $w$ & Stability \\
\hline \multirow{2}{*}{-1} & $>1 / 3$ & -1 & $>0$ & $-1<w<1 / 3$ & center \\
\cline { 3 - 5 } & & 1 & $>0$ & $w<-1$ & saddle \\
\cline { 4 - 5 } & & & & $w>1 / 3$ & \\
\hline \multirow{2}{*}{1} & $>1 / 3$ & -1 & $<0$ & $w<-1$ & \multirow{2}{*}{ saddle } \\
\cline { 4 - 5 } & & & & $w>1 / 3$ & \\
\cline { 3 - 5 } & & 1 & $<0$ & $-1<w<1 / 3$ & centre \\
\hline
\end{tabular}

thus providing new interesting results. The solutions found display stability conditions rather different from those of the corresponding solutions in closed models and from the stability properties of the standard ES solution of GR.

In the case of LQC gravitational modifications to the Friedmann equations, a negative curvature index allows a neutrally stable static solution with $\Lambda<\kappa \rho_{c}$ and $w<-1$, in contrast to the GR case. In particular the LQC static solution exists and is stable in the case of positive values of the cosmological constant as long as $\Lambda<\kappa \rho_{c}$.

In the case of HL gravity two static solutions are found. The inclusion of the negative curvature index leads to a static solution ( $H L 1)$ with negative cosmological constant and vanishing energy density which is neutrally stable against homogeneous perturbations. Furthermore, a negative curvature index allows a static solution ( $H L 2)$ which can be either a saddle, for $w<-1$ and $w>1 / 3$, or a center for $-1<w<1 / 3$. 
As already observed in the frameworks of different modified models [27, 28, 32, 34], the regime of infinite cycles about the center fixed points must be eventually broken in order to enter the current expanding universe phase. To this aim a further mechanism is needed, whose analysis is beyond the scope of this paper.

\section{Acknowledgements}

The first named author would like to thank to C. Böhmer, F. Lobo, R. Maartens and K. Vandersloot for useful discussions. The authors are grateful to G. Vilasi for his thoughtfull advices and encouragement. This work is partially supported by Agenzia Spaziale Italiana (ASI) through the WP5000 project, by the Italian Ministero Istruzione Università e Ricerca (MIUR) through the PRIN 2008 grant and by the INFN-MICINN collaboration.

\section{References}

[1] Ashtekar A., Pawlowski T., Singh P. and Vandersloot K., Loop Quantum Cosmology of $k=1$ FRW Models, Phys. Rev. D 75 (2007) 024035.

[2] Barrow J., Ellis G., Maartens R. and Tsagas C., On the Stability of the Einstein Static Universe, Class. Quant. Grav. 20 (2003) L155-L164.

[3] Barrow J. and Tsagas C., On the Stability of Static Ghost Cosmologies, Class. Quant. Grav. 26 (2009) 195003.

[4] Böhmer C., The Einstein Static Universe with Torsion and the Sign Problem of the Cosmological Constant, Class. Quant. Grav. 21 (2004) 1119-1124.

[5] Böhmer C., Hollenstein L. and Lobo F., Stability of the Einstein Static Universe in $f(R)$ Gravity, Phys. Rev. D 76 (2007) 084005.

[6] Böhmer C. and Lobo F., Stability of the Einstein Static Universe in Modified Gauss-Bonnet Gravity, Phys. Rev. D 79 (2009) 067504.

[7] Böhmer C. and Lobo F., Stability of the Einstein Static Universe in IR Modified Hořava Gravity, arXiv:0909.3986 [gr-qc].

[8] Böhmer C., Hollenstein L., Lobo F. and Seahra S., Stability of the Einstein Static Universe in Modified Theories of Gravity, arXiv:1001.1266 [gr-qc].

[9] Bojowald M., Loop Quantum Cosmology, Living Rev. Rel. 11 (2008) 4.

[10] Canonico R., Parisi L., Stability of the Einstein Static Universe in Open Cosmological Models, Phys. Rev. D 82 (2010) 064005.

[11] Carloni S., Elizalde E. and Silva P., An Analysis of the Phase Space of Horava-Lifshitz Cosmologies, Class. Quant. Grav. 27 (2010) 045004. 
[12] Clarkson C. and Seahra S., Braneworld Resonances, Class. Quant. Grav. 22 (2005) 3653-3688.

[13] Clifton T. and Barrow J., The Existence of Gödel, Einstein and de Sitter Universes, Phys. Rev. D 72 (2005) 123003.

[14] Czuchry E., The Phase Portrait of a Matter Bounce in Horava-Lifshitz Cosmology, arXiv:0911.3891 [hep-th].

[15] Eddington A., On the Instability of Einstein's Spherical World, Mon. Not. Roy. Astron. Soc. 90 (1930) 668-688.

[16] Ellis G. and Maartens R., The Emergent Universe: Inflationary Cosmology with no Singularity, Class. Quant. Grav. 21 (2004) 223-232.

[17] Ellis G., Murugan J. and Tsagas C., The Emergent Universe: An Explicit Construction, Class. Quant. Grav. 21 (2004) 233-249.

[18] Gergely L. and Maartens R., Brane-world Generalizations of the Einstein Static Universe, Class. Quant. Grav. 19 (2002) 213-222.

[19] Ghodsi A and Hatefi E., Extremal Rotating Solutions in Horava Gravity, Phys. Rev. D 81 (2010) 044016.

[20] Goheer N., Goswami R. and Dunsby P., Dynamics of $f(R)$-cosmologies Containing Einstein Static Models, Class. Quant. Grav. 26 (2009) 105003.

[21] Goswami R., Goheer N. and Dunsby P., Existence of Einstein Static Universes and their Stability in Fourth-Order Theories of Gravity, Phys. Rev. D. 78 (2008) 044011.

[22] Gruppuso A., Roessl E. and Shaposhnikov M., Einstein Static Universe as a Brane in Extra Dimensions, JHEP 011 (2004) 0408.

[23] Hawking S. and Ellis G., The Large Scale Structure of Space-Time, Cambridge Univ. Press, Cambridge, 1973.

[24] Horava P., Quantum Gravity at a Lifshitz Point, Phys. Rev. D 79 (2009) 084008.

[25] Horava P., Membranes at Quantum Criticality, JHEP 0903 (2009) 020.

[26] Leon G. and Saridakis E., Phase-Space Analysis of Horava-Lifshitz Cosmology, JCAP 0911 (2009) 006.

[27] Lidsey J. and Mulryne D., A Graceful Entrance to Braneworld Inflation, Phys. Rev. D 73 (2006) 083508.

[28] Lidsey J., Mulryne D., Nunes N. and Tavakol R., Oscillatory Universes in Loop Quantum Cosmology and Initial Conditions for Inflation, Phys. Rev. D 70 (2004) 063521.

[29] Lu H., Mei J. and Pope C., Solutions to Horava Gravity, Phys. Rev. Lett. 103 (2009) 091301. 
[30] Minamitsuji M., Classification of Cosmology with Arbitrary Matter in the Hořava-Lifshitz Theory, Phys. Lett. B 684 (2010) 194-198.

[31] Mukherjee S., Paul B., Dadhich N., Maharaj S. and Beesham A., Emergent Universe with Exotic Matter, Class. Quant. Grav. 23 (2006) 6927-6933.

[32] Mulryne D., Tavakol R., Lidsey J. and Ellis G., An Emergent Universe from a Loop, Phys. Rev. D 71 (2005) 123512.

[33] Parisi L., Dynamical Systems Techniques in Cosmology. An Example: LQC and the Einstein Static Universe, J. Geom. Symm. Phys. 14 (2009) 67-83, (see also Geometry, Integrability and Quantization X, Avangard Press, Sofia 2009, pp 211-226).

[34] Parisi L., Bruni M., Maartens R. and Vandersloot K., The Einstein Static universe in Loop Quantum Cosmology, Class. Quant. Grav. 24 (2007) 62436254.

[35] Park M., The Black Hole and Cosmological Solutions in IR Modified Horava Gravity, JHEP 0909 (2009) 123.

[36] Seahra S. and Böhmer C., Einstein Static Universes are Unstable in Generic $f(R)$ Models, Phys. Rev. D 79 (2009) 064009.

[37] Seahra S., Clarkson C. and Maartens R., Delocalization of Brane Gravity by a Bulk Black Hole, Class. Quant. Grav. 22 (2005) L91-L102.

[38] Son E. and Kim W., Smooth Cosmological Phase Transition in the HoravaLifshitz Gravity, arXiv:1003.3055 [gr-qc].

[39] Vandersloot K., Loop Quantum Cosmology and the $k=-1$ RW Model, Phys. Rev. D 75 (2007) 023523.

[40] Wang A. and Wu Y., Thermodynamics and Classification of Cosmological Models in the Horava-Lifshitz Theory of Gravity, JCAP 0907 (2009) 012.

[41] Wu P. and Yu H., Emergent Universe from the Hovava-Lifshitz Gravity, Phys. Rev. D 81 (2010) 103522.

[42] Zhang K., Wu P. and Yu H., The Stability of Einstein Static Universe in the DGP Braneworld, Phys. Lett. B 690 (2010) 229-232.

Dipartimento di Fisica "E. R. Caianiello"

Università di Salerno

I-84084 Fisciano (Sa)

INFN Sezione di Napoli, GC di Salerno

via Ponte Don Melillo

I-84084 Fisciano (Sa), ITALY

Luca Parisi - E-mail address: parisi@sa. infn. it

Rosangela Canonico - E-mail address: canonicodsa. infn. it 